\title{
Hexagonal PWR-Core Modeling and Simulation with Application of NECP- Bamboo
}

\author{
Cheng Zhang, Liangzhi Cao, Yunzhao Li", Guowei Hua \\ School of Nuclear Science and Technology, Xi'an Jiaotong University, \\ 28 West Xianning Road, Xi'an, Shaanxi 710049, China \\ *yunzhao@xjtu.edu.cn
}

\begin{abstract}
In this paper, the modeling and simulation of the PWRs loaded with hexagonal fuel assemblies has been implemented with the NECP-Bamboo code. NECP-Bamboo, consisting of a 2D lattice code named Bamboo-Lattice and a 3D steady-state core code named Bamboo-Core, was primitively designed for the PWRs loaded with the rectangular fuel assemblies. As the capability extension for PWRs with hexagonal fuel assemblies, four aspects of improvement have been implemented in NECP-Bamboo. Firstly, the Constructive Solid Geometry (CSG) has been implemented in Bamboo-Lattice for the lattice modeling. Secondly, the explicit modeling of the reflector assembly has been applied to provide more reliable few-group constants, compared with the conventional 1D model for the reflector assembly. Thirdly, the assembly-homogenization capability has been extended to the hexagonal assembly. Fourthly, the diffusion solver in Bamboo-Core based on the Variational Nodal Method (VNM) has been extended to handle hexagonal geometry. With application of the capability-extended NECPBamboo, the modeling and simulations for the VVER-1000 benchmark loaded with MOX fuel has been implemented. It can be observed that the numerical results provided by NECPBamboo can agree well with corresponding results by the Monte-Carlo code.
\end{abstract}

KEYWORDS: PWR, hexagonal core, NECP-Bamboo, assembly homogenization

\section{INTRODUCTION}

In China, there are four VVER units under operation. As the requirement proposed by the fuel management and technique supports for these VVER reactors, the reactor-physics code is required. For a long time, the KASKAD code developed by the Kurchatov Institute has been used for the modeling and simulations for the VVER reactor in China. However, the conventional methodologies have been used in most of the codes employed for the hexagonal PWR-cores [1], including collision probability method coupling neutron current method for solving the transport equation on the assembly level. Therefore, it is important to develop the newly code for VVER, with application of the most advanced methodologies proposed in recent years.

The PWR-core fuel management package named NECP-Bamboo [2] [3], was primitively designed for the PWRs loaded with the rectangular fuel assemblies, which consisted of a 2D lattice code Bamboo-Lattice and a 3D steady-state core code Bamboo-Core. In Bamboo-Lattice, modular MOC was employed to conduct heterogeneous transport calculation [4] and hence directly used for the assembly homogenization without the cell homogenization. In Bamboo-Core, the Variational Nodal Method was employed to solve the $3 \mathrm{D}$ whole-core diffusion equation. 
In order to simulate the PWR-cores like VVER, both the lattice and core codes of NECP-Bamboo have to be improved. As the improvement for the lattice code, a new MOC solver based on the hexagonal geometry, has been developed, in which the constructive solid geometry (CSG) method was employed to describe complex hexagonal geometry. Also the capabilities for the detailed reflector modeling and homogenization method for the hexagonal assembly have been extended in the lattice code. As improvement for the core code, the diffusion solver has been extended to handle the hexagonal assembly and core. After the improvements, the NECP-Bamboo code has been used to perform the modeling and simulation for the VVER-1000 benchmark, comparing the results proposed by the other researches. Moreover, the 2D wholecore simulation for the $\mathrm{S} 3$ state has been established as the references.

\section{Methods and Theories}

The conventional 'two-step' scheme with the assembly homogenization was applied in the NECP-Bamboo code. As simple introductions for the methodologies applied: in Bamboo-Lattice, the sub-group method was adopted to conduct resonance calculation, the CRAM method was employed to solve the Bateman equation and the predictor-correlator method was applied for the burnup calculation; For the core code, besides the VNM diffusion calculation, functions such as neutronic-thermohydraulic coupling, critical boron concentration search and reactivity coefficient calculation have already been achieved. Most of these methodologies can be applied to both the rectangular and hexagonal assembly and core. In order to simulate the PWRs loaded with hexagonal assemblies, the main issue is to extend the geometry-modeling capability to the hexagonal assembly in the lattice code and hexagonal configuration in the core code. Therefore, the improvements towards the VVER simulations have been focused on in this paper. In this section, four aspects of improvements of NECP-Bamboo would be introduced in detail.

\section{1. the CSG method for assembly modeling}

The hexagonal Bamboo-Lattice was based on a hexagonal modular MOC solver [5], in which CSG method was adopted for the geometry description. In this way, the lattice code Bamboo-Lattice would be capable to deal with arbitrary geometry within each assembly if their frames were hexagonal. The improvement of geometry description made it possible to treat complex assembly geometry directly, especially for reflectors.

The CSG method was widely used in the Monte-Carlo codes because of its strong ability of geometry modeling. The MOC method has the advantage for different geometries as well. However, most of the MOC-based lattice codes for PWR were designed for the structural geometry like the rectangular assembly. However, if the fuel pins in the assembly were not distributed regularly, these conventional lattice codes would fail to perform the modeling and simulation. Based on the notable advantages of CSG for the geometry modeling, , the Bamboo-Lattice code was improved with the CSG modelling method for the hexagonal assembly.

In the improved Bamboo-Lattice for the hexagonal assembly, the geometry modeling is defined as following. Firstly, a series of surfaces were defined. Then, with these surfaces as the boundaries, several cells were defined. Finally, the assembly were generated, with the defined cells.

Besides the basic nested structure of geometry description, Bamboo-Lattice had the functions of translation and rotation as well. What's more, three array types are optional. The first type was square lattice like the fuel pin arrangement in traditional rectangular PWR assemblies. The second one was hexagonal lattice for assemblies in hexagonal PWR cores like VVER. The third one was circular cluster array for constructing some cluster-type fuel assemblies, used in CANDU and RBMK reactors. 
With all these functions mentioned above, the Bamboo-Lattice code has the modeling capability to deal with the complex geometries of the hexagonal assembly. For the fuel pin in VVER-1000 benchmark loaded with MOX fuel, the flat source regions (FSRs) were divided as shown in Figure 1. The division of the irregular regions near the assembly boundary are as shown in Figure 2. The regular hexagonal fuel pins and the irregular regions were arranged in hexagonal lattice, forming a VVER-1000 fuel assembly.

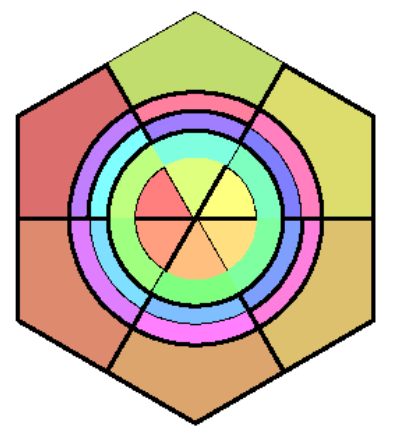

Figure 1. FSRs division of a fuel pin.

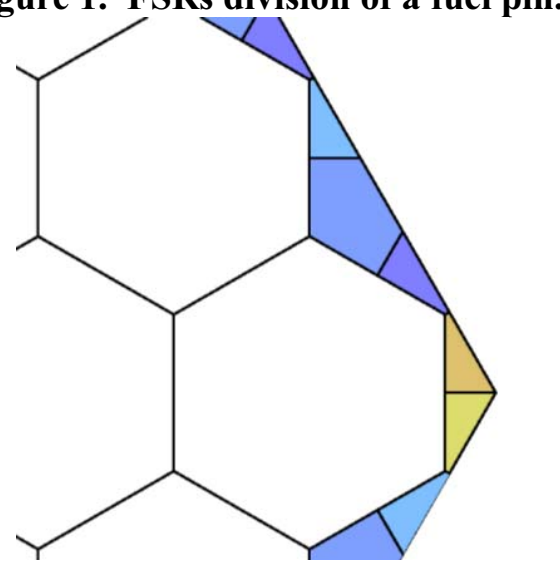

Figure 2. FSRs division near fuel assembly.

\section{2. the Reflector modelling}

The accuracy of the few-group constants for the reflectors are important for the "two-step" scheme, as it has notable effects on the power distributions. Notably different with the core loaded with rectangular assembly, there are more surfaces in the core loaded with the hexagonal assembly, which results in more complex intersections between nearby assemblies. In this case, the conventional one-dimension model for the reflector is not suitable any more for the hexagonal core. Therefore, it becomes an issue how to establish a reliable reflector modeling for the hexagonal core.

Using Bamboo-Lattice, the reflector modeling for the VVER-1000 core is as shown in Figure 3. Considering the symmetry of the reflector geometry, there are 5 reflector nodes (in blue boundaries) in total. The first two are covered in one reflector model with a red hexagon boundary.

This reflector model differs from the origin 'super-assembly' model, which covers only one fuel node and one reflector node. There are two considerations here. First, a hexagonal reflector node shares more interfaces with fuel than a Cartesian one. Second, the new model covers bigger volume thus the model 
boundary is far away from the surfaces of reflector nodes where discontinuity factors are calculated. This practice weakens the approximation of the boundary condition of the whole reflector model.

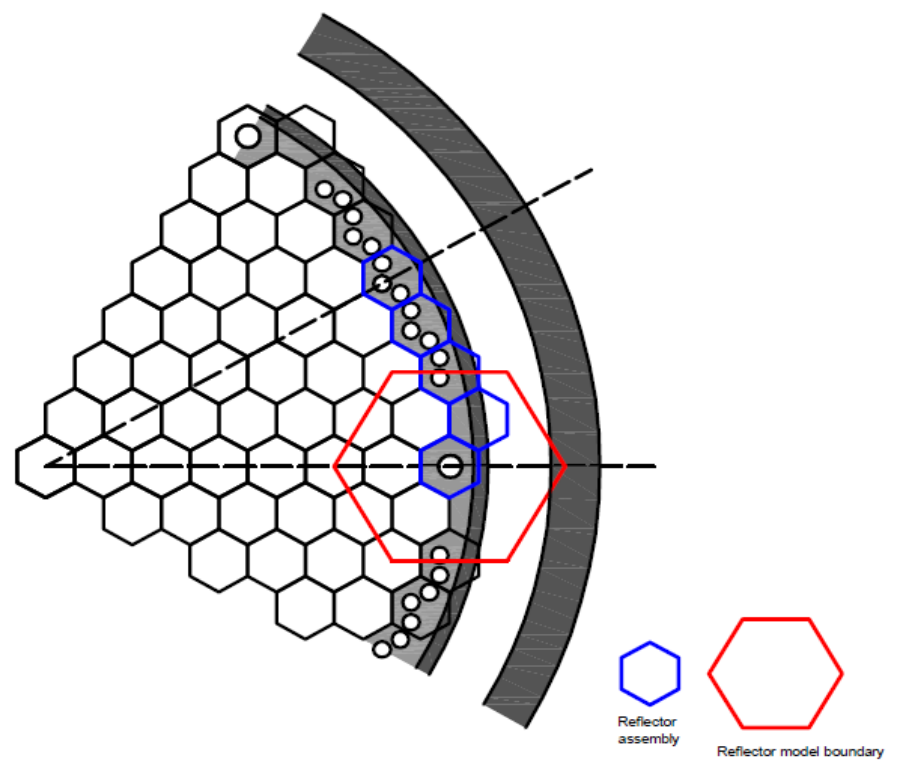

Figure 3. Reflector model.

\subsection{Hexagonal fuel assembly homogenization}

With the neutron fluxes and multigroup cross sections, the generalized equivalence theory has been applied to calculate the assembly-homogenized few-group constants for the hexagonal assembly. To maintain the reaction-rate balance, the homogeneous few-group constants should be calculated as below:

$$
\sum_{x, i, g}^{h o m}=\frac{\sum_{h \in g} \int_{V_{i}} \sum_{x, h}^{\text {het }}(\boldsymbol{r}) \phi_{h}^{\text {het }}(\boldsymbol{r}) d \boldsymbol{r}}{\int_{V_{i}} \phi_{g}^{h \mathrm{et}}(\boldsymbol{r}) d \boldsymbol{r}} \quad x=a, f, s \cdots
$$

Where:

$\sum_{x, h}^{h e t}$ is the cross section used by the transport calculation of the $\mathrm{h}_{\text {th }}$ group;

$\phi_{h}^{\text {het }}$ is the heterogeneous flux of the $\mathrm{h}_{\mathrm{th}}$ group.

The diffusion coefficient has many sources, such as the leakage coefficient in the process of leakage correction. In Bamboo-Lattice, the diffusion coefficient is calculated from the transport cross section $\Sigma_{\text {tr }}$. Among the transport correction methods, the inflow correction is recommended:

$$
\Sigma_{t r, g}=\Sigma_{t, g}-\frac{\sum_{g^{\prime}} \Sigma_{s 1, g^{\prime} \rightarrow g} \phi_{1, g^{\prime}}}{\phi_{1, g}}
$$

Where:

$\Sigma_{\mathrm{t}, \mathrm{g}}$ is the total cross section of the $\mathrm{g}_{\mathrm{th}}$ group;

$\Sigma_{s 1, g^{\prime} \rightarrow g}$ is the first-order scattering cross section from the $\mathrm{g}_{\text {th }}^{\prime}$ group to the $\mathrm{g}_{\text {th }}$ group;

$\phi_{1, g}$ is the first-order flux momentum of the $\mathrm{g}_{\text {th }}$ group. 
The multi-group diffusion coefficient can be derived from Fick's law:

$$
D_{h}=\frac{1}{3 \Sigma_{t r, h}}
$$

The few-group diffusion coefficient is merged by the flux-volume weight based on the multigroup ones:

$$
D_{g}=\frac{\sum_{h \in g} \int_{V} D_{h} \cdot \phi_{h}(r) d r}{\sum_{h \in g} \int_{V} \phi_{h}(r) d r}
$$

To keep the balance between the leakage of heterogeneous and homogeneous calculations, the discontinuity factor is required and characterized as:

$$
f_{i, g}^{s}=\frac{\phi_{i, g}^{s, h e t}}{\phi_{i, g}^{s, h o m}}
$$

For the fuel assemblies, homogeneous surface flux $\phi_{i, g}^{s, h o m}$, can be approximately replaced by heterogeneous volume-averaged flux $\phi_{g}^{A, h \text { et }}$

$$
f_{i, g}^{s}=\frac{\phi_{i, g}^{s, h e t}}{\phi_{g}^{A, h \mathrm{et}}}
$$

While for the reflector regions, considering the net neutron current near the nodal boundary could not be neglected, the zero-net-current approximation is not suitable. The homogeneous surface flux should be obtained by solving a single-node diffusion problem, in which the few-group constants come from the homogenization of the corresponding region. The boundary condition of the diffusion problem ought to be set according to the net current computed after the heterogeneous transport calculation. The method adopted to solve the diffusion equation should be consistent with the diffusion solver in the core code. In the latest version of Bamboo-Lattice, Variational Nodal Method (VNM) and Analytical Function Expansion Method (AFEN) are optional for the solution of diffusion equation mentioned above.

\subsection{Extended VNM solver}

Compared with the origin version, the latest Bamboo-Core was equipped with a hexagonal VNM solver to deal with PWRs like VVER. VNM is mature in theory and can guarantee the nodal neutron balance equation with good computational accuracy. Based on the theory, a hexagonal VNM diffusion solver named NECP-Violet [6] [7] has been developed. The solver has been strictly verified by a series of diffusion benchmarks. The results of 2D IAEA benchmark with different boundary conditions are listed below. 
Table I. Results of some diffusion benchmarks.

\begin{tabular}{|c|c|c|c|c|}
\hline \multirow[b]{2}{*}{ Benchmark } & \multirow[b]{2}{*}{ Code } & \multirow[b]{2}{*}{$\begin{array}{l}\text { Bias of } \\
\text { keff } \\
(\mathrm{pcm})\end{array}$} & \multicolumn{2}{|c|}{ Assembly power } \\
\hline & & & $\begin{array}{l}\text { Maximum } \\
\text { relative } \\
\text { error } / \%\end{array}$ & $\begin{array}{c}\text { Minimum } \\
\text { relative } \\
\text { error } / \%\end{array}$ \\
\hline \multirow{4}{*}{$\begin{array}{l}\text { IAEA with the } \\
\text { albedo of } \\
0.5 \text { (no } \\
\text { reflector) }\end{array}$} & ANC-H & 6 & 0.5 & - \\
\hline & $\begin{array}{c}\text { SIXTUS- } \\
2 \\
\end{array}$ & 83.7 & -1.54 & 0.45 \\
\hline & DIF3D-N & 173.6 & 4 & - \\
\hline & $\begin{array}{l}\text { NECP- } \\
\text { Violet }\end{array}$ & 1 & 0.03 & 0 \\
\hline \multirow{4}{*}{$\begin{array}{l}\text { IAEA with the } \\
\text { albedo of } \\
0.125 \text { (no } \\
\text { reflector) }\end{array}$} & ANC-H & -7 & 0.8 & - \\
\hline & $\begin{array}{c}\text { SIXTUS- } \\
2\end{array}$ & 137.2 & 4.3 & 1.61 \\
\hline & DIF3D-N & 208.2 & 4.3 & - \\
\hline & $\begin{array}{l}\text { NECP- } \\
\text { Violet }\end{array}$ & 1 & 0.01 & 0 \\
\hline \multirow{4}{*}{$\begin{array}{l}\text { IAEA with the } \\
\text { albedo of } \\
0.5 \text { (with } \\
\text { reflector) }\end{array}$} & ANC-H & -2 & 0.7 & - \\
\hline & $\begin{array}{c}\text { SIXTUS- } \\
2 \\
\end{array}$ & 22.3 & 1.52 & 0.46 \\
\hline & DIF3D-N & -338.8 & 18.1 & - \\
\hline & $\begin{array}{l}\text { NECP- } \\
\text { Violet }\end{array}$ & 1 & 0.03 & 0 \\
\hline \multirow{4}{*}{$\begin{array}{l}\text { IAEA with the } \\
\text { albedo of } \\
0.125 \text { (with } \\
\text { reflector) }\end{array}$} & ANC-H & 7 & 0.5 & - \\
\hline & $\begin{array}{c}\text { SIXTUS- } \\
2 \\
\end{array}$ & 0.8 & 2.13 & 0.7 \\
\hline & DIF3D-N & -409.5 & 20.2 & - \\
\hline & $\begin{array}{l}\text { NECP- } \\
\text { Violet }\end{array}$ & 1 & 0.01 & 0 \\
\hline
\end{tabular}

\section{NUMERICAL RESULTS}

The VVER-1000 MOX computational benchmark [8] was applied to verify the modeling ability of NECPBamboo for the PWR loaded with the hexagonal assembly. There are 6 core states proposed for the benchmark, with different fuel temperatures, moderator temperatures and boron concentrations. In this chapter, the state S3, which stands for the cold state, was chosen to be simulated. At core state S3, all components in the core remain the temperature of $300 \mathrm{~K}$ and the boron concentration is $2800 \mathrm{ppm}$. In this chapter, all the references are results of one-step calculation by Monte Carlo code.

There are two different kinds of assemblies (UOX and MOX assemblies) in the VVER-1000 core. the configuration of the fuel assemblies with different burnup is as shown in Figure 4. Combined with the fuel types and different burnups, there are 7 different kinds of assemblies loaded in the core, including UOX_0, UOX_15, UOX_32, UOX_40, MOX_0, MOX_17 and MOX_33. Take one assembly as example, the UOX_40 stands for the UOX assembly with burn-up $40 \mathrm{GWd} / \mathrm{tU}$. For the assembly modeling and simulations with the lattice code, the $k_{\mathrm{inf}}$ by Bamboo-Lattice and corresponding reference values are 
compared, as shown in Table II. It can be observed that the assembly $k_{\text {infs }}$ by Bamboo-Lattice agree well with corresponding reference values.

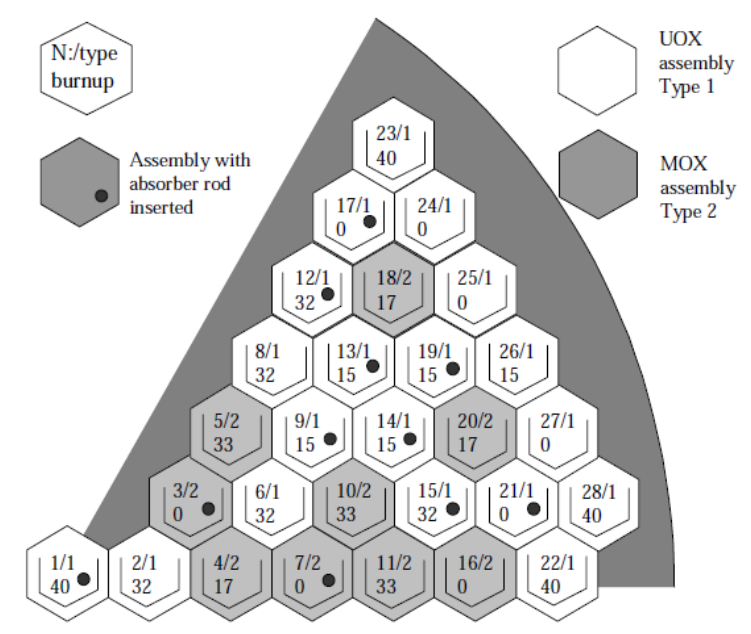

Figure 4. Assembly arrangement in the VVER-1000 core.

Table II. Effective multiplication factors of the 7 assemblies.

\begin{tabular}{|c|c|c|c|}
\hline Assembly & Reference & Bamboo-Lattice & Bias/pcm \\
\hline UOX_0 & 1.03476 & 1.03499 & 23 \\
\hline UOX_15 & 0.96149 & 0.96302 & 153 \\
\hline UOX_32 & 0.84827 & 0.85007 & 180 \\
\hline UOX_40 & 0.80043 & 0.79843 & -200 \\
\hline MOX_0 & 1.07211 & 1.07144 & -67 \\
\hline MOX_17 & 0.96808 & 0.96877 & 70 \\
\hline MOX_33 & 0.85534 & 0.85571 & 37 \\
\hline
\end{tabular}

Based on the verifications for the assembly modeling and simulations, the $2 \mathrm{D}$ whole-core modeling and simulations have been implemented by Bamboo-Core, with the results shown in Table III and Figure 5. . The bias of $k_{\text {eff }}$ is $199 \mathrm{pcm}$ and the relative biases of the assembly fission rate are mostly less than $2 \%$. It can be concluded from the simulation of VVER-1000 S3 state core that NECP-Bamboo has the capacity to handle PWRs with hexagonal assembly arrangement.

Table III. Effective multiplication factor of S3 state core.

\begin{tabular}{|c|c|c|c|}
\hline & Reference & Bamboo-Core & Bias $/ \mathrm{pcm}$ \\
\hline$k_{\text {eff }}$ & 0.93452 & 0.93651 & 199 \\
\hline
\end{tabular}




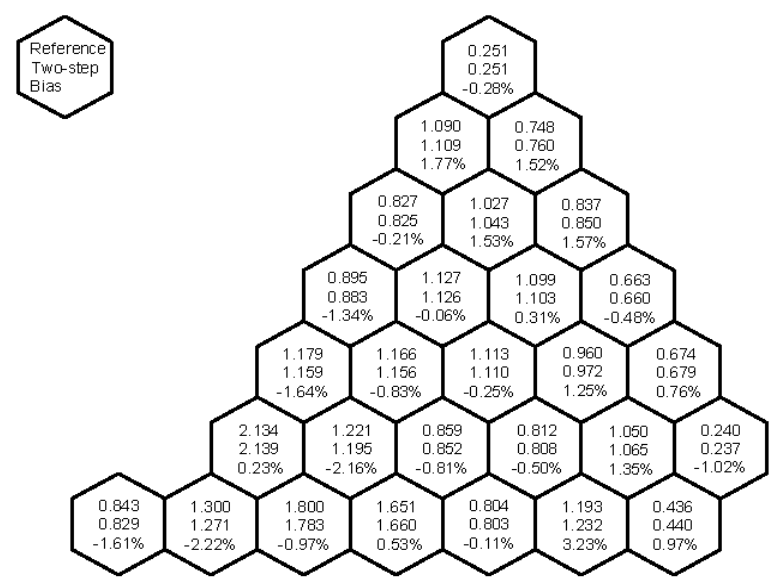

Figure 5. Assembly fission rate distribution of VVER-1000 core at S3 state.

\section{CONCLUSIONS}

It could be concluded from the previous chapter that both the results of Bamboo-Lattice and Bamboo-Core meet well with the reference. The biases of eigenvalue of single-assembly cases are less than $200 \mathrm{pcm}$. The bias of $k_{\text {eff }}$ of the S3 state core is $199 \mathrm{pcm}$ and the biggest bias of assembly fission rate is $3.23 \%$. In one word, the newly developed NECP-Bamboo has great advantages on geometry description and its results of the two-step simulation of the VVER-1000 benchmark have great agreements with reference. Thus, geometry description of NECP-Bamboo has been extended to hexagon and its capacity to simulate hexagonal cores like VVER has been proved.

\section{ACKNOWLEDGMENTS}

This work is financially supported by the National Natural Science Foundation of China (11975181).

\section{REFERENCES}

1. IAEA. In-core fuel management code package validation for WWERs, IAEA-TECDOC-847, International Atomic Energy Agency, 1995.

2. Y. Li, B. Zhang, et al., "Development and Verification of PWR-Core Fuel Management Calculation Code System NECP-Bamboo: Part I Bamboo-Lattice," Nuclear Engineering and Design, 335, pp.432440 (2018).

3. Yang W, Wu HC, Li YZ, et al. "Development and verification of PWR: Core nuclear design code system NECP-Bamboo, Part II: Bamboo-Core," Nuclear Engineering and Design, 337, pp.279-290 (2018).

4. C. Tian, Y. Zheng, et al., "Neutron transport study based on assembly modular ray tracing MOC method," Nuclear Power Engineering, VOL 36, pp. 157-161, June 15, (2015).

5. Cho JY et al., "Whole core Transport Calculation Employing Hexagonal Modular Ray Tracing and CMFD Formulation". J Nuclear Science Technology, 45, p.740-751(2008).

6. $\mathrm{Li} \mathrm{Y,} \mathrm{Li} \mathrm{Z,} \mathrm{et} \mathrm{al.} \mathrm{Improved} \mathrm{variable-Transformation} \mathrm{algorithm} \mathrm{for} \mathrm{the} \mathrm{hexagonal} \mathrm{variational} \mathrm{nodal}$ method[J]. Transactions of American Nuclear Society, 2016, 115: 520 522.

7. Li Z, Wu H, Li Y, et al. Application of the Symmetry Group Theory to the VNM Response Matrices Simplification[C]. Reactor Physics Asia, 2017, Chengdu, China.

8. NEA/NSC/DOC 17, 2005. VVER-1000 MOX Core Computational Benchmark. Nuclear Energy Agency, Organization for Economic Co-operation and Development. 